\title{
Signal-to-noise ratio of the MEG signal after preprocessing
}

\section{H I G H L I G H T S}

- The signal-to-noise ratio of event-related fields is used to evaluate the effectiveness of various preprocessing algorithms for magnetoencephalography data.

- Signal Space Separation algorithms provide approximately a $100 \%$ increase in signal to noise ratio.

- Epoch-based artifact rejection and decomposition methods such as independent component analysis yielded a signal to noise ratio increase of 5-10\% and $35 \%$ respectively. The use of decomposition methods seems advisable.

- The evaluation of the signal-to-noise ratio increase can help to guide the choice of preprocessing methods.

\begin{abstract}
A B S T R A C T
Background: Magnetoencephalography (MEG) provides a direct measure of brain activity with high combined spatiotemporal resolution. Preprocessing is necessary to reduce contributions from environmental interference and biological noise.

New method: The effect on the signal-to-noise ratio of different preprocessing techniques is evaluated. The signal-to-noise ratio (SNR) was defined as the ratio between the mean signal amplitude (evoked field) and the standard error of the mean over trials.

Results: Recordings from 26 subjects obtained during and event-related visual paradigm with an Elekta MEG scanner were employed. Two methods were considered as first-step noise reduction: Signal Space Separation and temporal Signal Space Separation, which decompose the signal into components with origin inside and outside the head. Both algorithm increased the SNR by approximately $100 \%$. Epoch-based methods, aimed at identifying and rejecting epochs containing eye blinks, muscular artifacts and sensor jumps provided an SNR improvement of 5-10\%. Decomposition methods evaluated were independent component analysis (ICA) and second-order blind identification (SOBI). The increase in SNR was of about $36 \%$ with ICA and $33 \%$ with SOBI.

Comparison with existing methods: No previous systematic evaluation of the effect of the typical preprocessing steps in the SNR of the MEG signal has been performed.

Conclusions: The application of either SSS or tSSS is mandatory in Elekta systems. No significant differences were found between the two. While epoch-based methods have been routinely applied the less often considered decomposition methods were clearly superior and therefore their use seems advisable.
\end{abstract}

\section{Introduction}

MEG allows obtaining spatiotemporal maps of brain activity with high resolution (Hamalainen et al., 1993; Hari and Salmelin,
2012) and it is increasingly being employed in basic and clinical neuroscience research. Given the small magnitude of the magnetic fields induced by the electrical currents produced by the neurons, extremely sensitive sensors are employed (Hamalainen et al., 1993).

In the present work we are concerned with the effectiveness of the preprocessing methods typically employed with MEG data in reducing unwanted signal components or artifacts.

A review of preprocessing methods for MEG is provided in Parkkonen (2010) and Gross et al. (2013). There are three types of artifact depending on their origin (Gross et al., 2013): System related artifacts due to noisy sensors, environmental artifacts such 
as noise from power lines, and physiological artifacts. The latter are typically caused by eye movements, eye blinks, cardiac and muscular activity and head movements.

System artifacts are reduced by rejecting/interpolating flat and very noisy channels and by filtering out line noise, for example with notch filters at the appropriate frequencies. The way to address environmental noise is manufacturer dependent. In Elekta (Helsinki, Finland) scanners two methods are used: Signal Space Separation (SSS) (Taulu et al., 2004, 2005) and temporal Signal Space Separation (tSSS) (Taulu and Simola, 2006). Both methods aim at decomposing the signal into contributions originating inside and outside the head and eliminating the latter. Other systems such as CTF/VSM (Coquitlam, BC, Canada), 4D Neuroimaging (San Diego, CA, USA) and Yokogawa (Tokyo, Japan) relay on an array of reference sensors located further away from the brain than the measurement sensors to project out this type of noise (Vrba and Robinson, 2001). Although SSS and tSSS are the methods of choice in Elekta systems for offline analysis, for real-time data visualization signal space projection (SSP) (Uusitalo and Ilmoniemi, 1997; Nolte and Curio, 1999; Parkkonen, 2010) is typically used. To use SSP, a principal components analysis (PCA) is performed using an empty room dataset to identify the subspace where external artifacts are reflected in sensor space. Typically 3-5 components representing most of the variance of the external artifacts are selected. Such components are stable over time unless the magnetic environment undergoes a drastic change. These components are then projected out from measurement data to reduce the contribution from external artifacts.

Two main types of, not mutually exclusive, methods exist to eliminate or reduce physiological artifacts. With epoch-based methods, epochs containing artifacts can be identified according, for instance, to the amplitude or spectral content of the signal. These epochs are then eliminated from the datasets. A second approach is to use decomposing methods, also called blind-source separation methods, such as independent component analysis (ICA) (Comon, 1994; Hyvrinen and Oja, 2000) or second order blind identification (SOBI) (Belouchrani et al,, 1997; Cardoso, 1998). These methods are less commonly employed. They have the advantage of eliminating components rather than epochs. Therefore, more data is preserved for further analysis. The disadvantage is that they are more time-consuming and less objective, as components typically need to be identified visually. Preprocessing with blind source separation has been shown to improve EEG-based classification between Mild Cognitive Impairment patients subsequently converting to Alzheimer's Disease and healthy controls (Cichocki et al., 2005).

Interference suppression is aided by the use of passive magnetically shielded rooms in MEG systems which provide shielding especially at low frequencies. Averaging increases the SNR as $\sqrt{N}$, where $N$ is the number of averaged trials, provided that the noise in the data is temporally uncorrelated from trial to trial, and trials are artifact-free. In addition, time-domain filtering of the data can help to improve the SNR as signals of interest tend to be located in the $1-100 \mathrm{~Hz}$ frequency band avoiding part of the environmental and physiological noise below this band and some of the system-noise above (Parkkonen, 2010).

In the present study the objective was to characterize the change in signal-to-noise ratio (SNR) after applying the most common artifact detection and artifact reduction algorithms during MEG data preprocessing. The SNR has been previously employed to assess the quality of preprocessing. For instance, auto-adaptive averaging methods have been proposed to decide which epochs to reject by optimizing the SNR of the event-related potentials (ERP) (Talsma, 2008). Similarly, performing a trimmed average has been shown to be advantageous over arithmetical averages in terms of SNRs of ERPs (Leonowicz et al., 2005). Along the same lines, wavelet filtering has been reported to improve the SNR of a particular ERP component, the N1 wave (Hu et al., 2010). In addition to the SNR, a related measure of noise, the variance in the ERP baseline, has been employed to compare the performance of different statistical thresholding schemes and supervised artifact rejection (Nolan et al., 2010).

In the present work, the SNR was defined as the ratio between the amplitude of the event-related field and its standard deviation across epochs, averaged over channels and latencies of interest. Datasets from a visual event-related paradigm under two experimental conditions were employed. The effect of the different preprocessing algorithms on the SNR was assessed.

\section{Methods}

\subsection{Datasets}

26 datasets from a visual event-related paradigm were considered. Recordings were performed with an Elekta MEG whole-head scanner at the Centre for Biomedical Technology, Technical University of Madrid, Spain. This scanner comprises 306 channels, of which, 204 are planar gradiometers and 102 magnetometers. In the present work we focused on the signal from the magnetometers after a preliminary analysis showed that the behavior with respect to SNR changes with preprocessing was similar for both types of sensors. Participants included in the present analysis were 13 healthy controls and 13 patients with Mild Cognitive Impairment. A delayed match-to-sample paradigm was employed. There were two experimental conditions comprising encoding, maintenance and recognition phases. Condition 1 consisted in the presentation of face stimulus for $1 \mathrm{~s}$, a $4 \mathrm{~s}$ maintenance phase, and a recognition phase were the same or a different face was presented for an additional second, and participants had to respond whether it was the same face as before. Condition 2 had the same structure except that an additional interference stimulus, also a face, was presented during the maintenance phase. The study was granted ethical approval by the review board of the Hospital Clínico San Carlos, Madrid, Spain. Unless otherwise stated results are obtained from the encoding phase of condition 1.

13 of the 26 datasets were recorded with continuously active position coils attached to the head of the participants. These allow estimating the position of the head during scanning by producing a high-frequency sinusoidal magnetic field.

\subsection{First-pass preprocessing}

Preprocessing with SSS and tSSS was carried out with the scanner manufacturer's software MaxFilter 2.1. Signal Space Separation (SSS) (Taulu et al, 2004) SSS exploits the fact that, following Maxwell equations for electromagnetism, magnetic sources inside and outside the sensor array project the signal to different subspaces within the sensor space. A series of spherical harmonic functions is used to expand the signal in this two subspaces and only the part arising form inside the sensor array is retained. One requirement of the SSS method is that accurate information about the geometry of the sensor array is provided. A limitation is that artifacts from sources near the sensor array such as metallic implants, stimulators and dental fillings will project to both the inside and outside subspace and are not properly eliminated.

To target these components an extension of the SSS method, termed temporal Signal Space Separation (tSSS) (Taulu and Simola, 2006), can be used. tSSS complements the SSS procedure by performing an ICA analysis separately on the inside and outside subsignals. Subsequently, temporal correlations between both subsets of ICA components are identified and inside components highly 


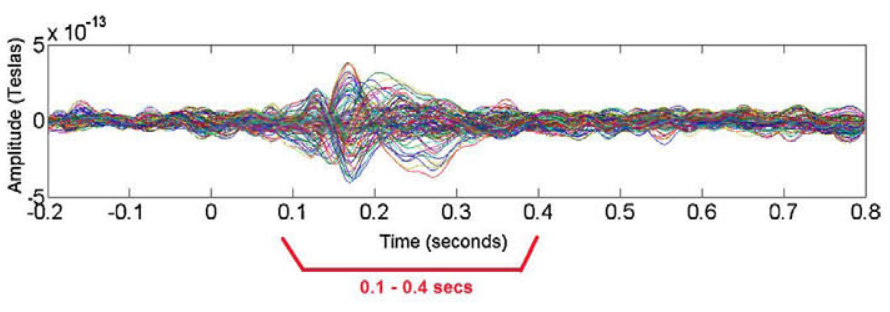

Fig. 1. Event-related fields from a representative dataset.

correlated with their outside counterparts are eliminated as they are likely to reflect artifacts contaminating both subspaces.

\subsection{Epoch-based artifact rejection}

Identification of artifact-contaminated epochs was carried out within the Fieldtrip toolbox (Oostenveld et al., 2011). Event related data was bandpass filtered between 1 and $40 \mathrm{~Hz}$ and baseline corrected with respect to a $[-250: 0] \mathrm{ms}$ time window. A notch filter at $50 \mathrm{~Hz}$ was also applied.

An example of event-related fields is provided in Fig. 1. A time-window of interest was defined from $100 \mathrm{~ms}$ to $400 \mathrm{~ms}$ after stimulus onset.

The Fieldtrip function $\mathrm{ft}_{\text {_artifact_}} z$ value was used to detect artifacts for epoch rejection. In this algorithm a $z$-score time-course is calculated for each channel by subtracting the mean and dividing by the standard deviation across samples. $z$-Values are then averaged across channels resulting in a time-course representing the global standardized deviation. A threshold is chosen for the global $z$-score so that a given fraction of epochs with deviations above threshold at any time point are discarded. Ocular, muscle and jump of artifacts are independently identified with different sets of parameters specifying subsets of channels to consider, filtering bands, type of padding and $z$-score calculation. The default parameter values were adopted as specified in Table 1 except for the cutoff values. A global cutoff value across recordings was chosen visually, with the goal of maximizing the rejection of epochs with outlier $z$-score values, while preserving the main mode of the distribution as much as possible.

\subsection{Component-based artifact reduction}

Two algorithms for component elimination were assessed. Independent components analysis (ICA) (Comon, 1994) and second order blind identification (SOBI) (Belouchrani et al,, 1997; Tang et al., 2005) as implemented in Fieldtrip (Oostenveld et al., 2011).

Both ICA and SOBI are blind source separation method. ICA attempts to decompose the signal into components that are maximally independent in the statistical sense. Since artifacts, such as cardiac and eye activity, tend to be independent of brain

Table 1

Parameter values adopted for epoch-based artifact rejection.

\begin{tabular}{|c|c|c|c|}
\hline & Jump & Muscle & EOG \\
\hline $\begin{array}{l}\text { Epoch } \\
\text { padding }\end{array}$ & $0.5 \mathrm{~s}$ & - & $0.3 \mathrm{~s}$ \\
\hline $\begin{array}{l}\text { Filter } \\
\text { padding }\end{array}$ & - & $0.3 \mathrm{~s}$ & $0.3 \mathrm{~s}$ \\
\hline $\begin{array}{l}\text { Artifact } \\
\text { padding }\end{array}$ & $0.5 \mathrm{~s}$ & $0.3 \mathrm{~s}$ & $0.1 \mathrm{~s}$ \\
\hline Linear filter & - & $\begin{array}{l}\text { Butterworth, order } 9 \\
(110-140 \mathrm{~Hz})\end{array}$ & $\begin{array}{l}\text { Butterworth, order } 4 \\
(1-15 \mathrm{~Hz})\end{array}$ \\
\hline $\begin{array}{l}\text { Non-linear } \\
\text { filter }\end{array}$ & $\begin{array}{l}\text { Median filter, } \\
\text { order } 9\end{array}$ & - & - \\
\hline $\begin{array}{l}\text { Cuttoff } \\
\text { value }\end{array}$ & 50 & 12 & 16 \\
\hline
\end{tabular}

activity, the two types of sources tend to be represented by non-overlapping sets of components, which allows for artifact elimination.

SOBI differs from ICA in that it exploits the temporally delayed statistical dependencies of the sources that putatively contribute to the recorder signal as it attempts to minimize the cross-correlations between one component at time $t$ and another component at later times. In contrast, ICA is only concerned with minimizing the instantaneous, zero-delay, statistical dependencies.

Both ICA and SOBI rely on the assumption that the topographies of the artifacts are stable across time and can be described with a limited number of spatial components, which is typically the case for eye and cardiac artifacts.

These methods require the visual inspection of both the topography and time-course of the obtained component to identify and eliminate those corresponding to eye and cardiac artifacts. In terms of computational time, although the selection of components by visual inspection only adds on the order of 2-5 min per dataset on a desktop computer, compared to the 2 min that takes to automatically compute the ICA/SOBI components, or the 5-10 min for SSS or tSSS, the need for interaction precludes running the whole analysis in batch mode.

Fig. 2 shows the eliminated ICA (left) and SOBI (right) components for a representative dataset. The components represent eye and cardiac (bottom component) artifacts.

\subsection{Signal-to-noise ratio (SNR)}

For a given channel and latency, the signal-to-noise ratio was defined as the ratio of the signal mean, $\bar{x}$ (event-related field) to its standard error $\sigma_{\bar{x}}$ across epochs.

$$
\begin{aligned}
& \operatorname{SNR}_{0}=\frac{\bar{x}}{\sigma_{\bar{x}}} \\
& \bar{x}=\frac{1}{N} \sum_{i=1}^{N} x_{i} \\
& \sigma_{\bar{x}}=\sqrt{\frac{\sum_{i=1}^{N}\left(x_{i}-\bar{x}\right)^{2}}{N}}
\end{aligned}
$$

where $N$ denotes the number of epochs, and index $i=1 \ldots N$ runs over samples. A global SNR was then obtained by averaging across the $20 \%$ of samples (combinations of channel and latency) with highest SNR selected from any channel and a time-window of interest in the range $[100: 400] \mathrm{ms}$ post stimulus onset. Defining the global SNR as an average over individual SNRs comprising all channels and time-sample combinations in the window of interest yielded qualitatively similar results (data not shown).

\section{Results}

Fig. 3 (left panels) shows the signal amplitude in sensor space averaged across the time-window of interest [100: 400] ms for unprocessed data and for data processed with SSS and tSSS for a representative recording. The right panels present the event related fields for the individual channels. It is apparent that first-pass preprocessing has a clear effect on the signal.

Fig. 4 shows the mean SNR across the 26 recordings before and after preprocessing with SSS and tSSS. Errorbars denote the standard error of the mean. The two preprocessing methods are associated with an approximately twofold increase in SNR. Table 2 provides the SNR values changes for recordings with and without continuous head position monitoring. The increase in SNR is similar for both types of recordings.

Next, we investigate the effect of SSS and tSSS in the different traditional frequency bands. Evoked activity characterizes 

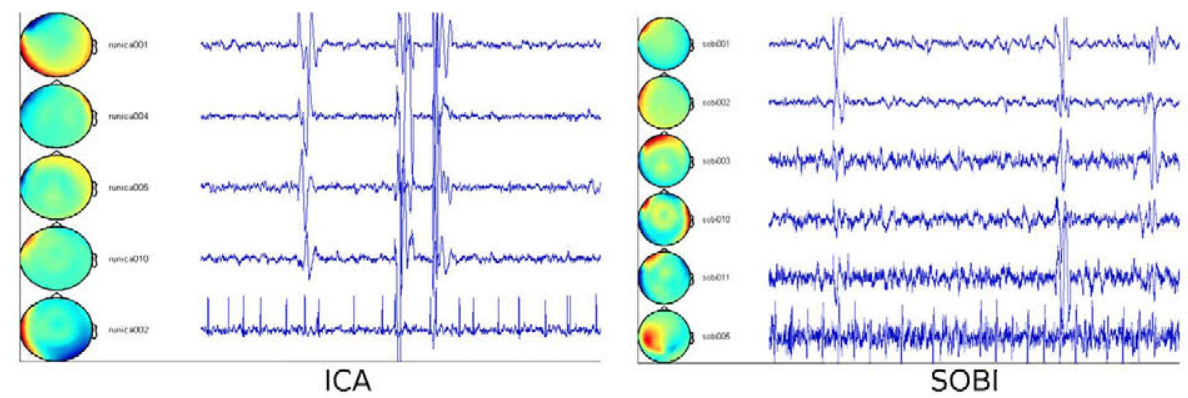

Fig. 2. Eliminated ICA (left) and SOBI (right) components for a representative dataset. Components represent eye and cardiac (bottom component) artifacts.

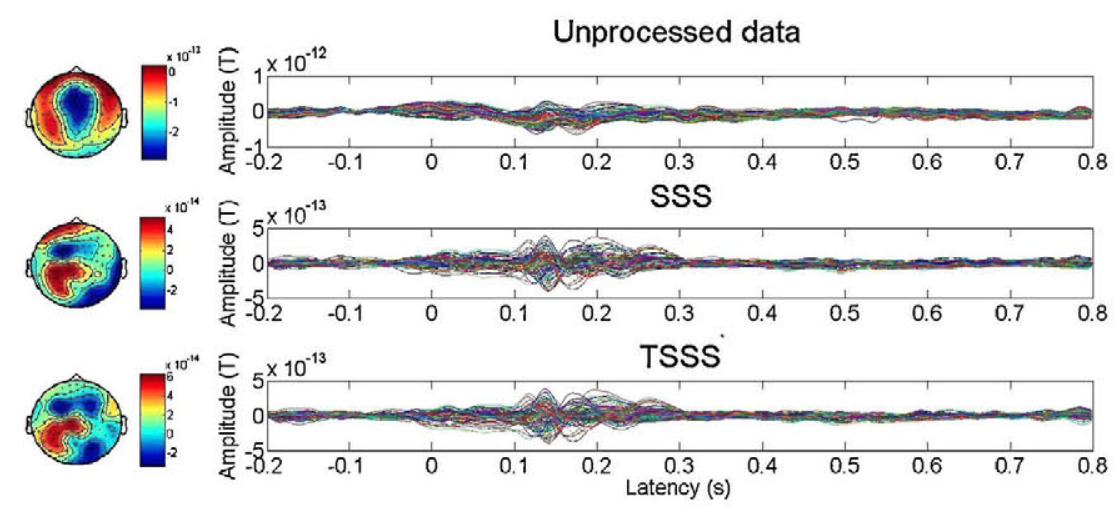

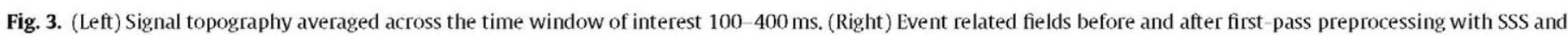
tSSS.

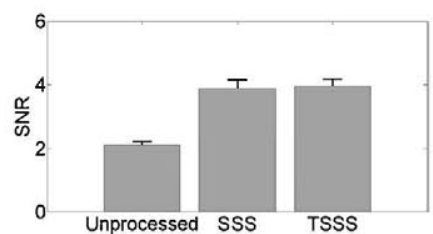

Fig. 4. Mean SNRs across the 26 recordings before and after preprocessing with SSS and tSSS. Errorbars denote the standard error of the mean.

signal changes phase-locked to the stimulus, while induced activity represents changes time-locked to the stimulus. Trials were bandpass-filtered in the following frequency bands: delta $(2-4 \mathrm{~Hz})$, theta $(4-8 \mathrm{~Hz})$, alpha $(8-15 \mathrm{~Hz})$, beta $(15-30 \mathrm{~Hz})$ and gamma $(30-60 \mathrm{~Hz})$. To obtain the induced activity the trial time-courses were squared before averaging across trials. The effect of SSS and tSSS in shown in Figs. 5 and 6 for evoked and induced activity respectively. Both methods provide similar results for the two types of activity. TSSS provides slightly higher SNR values for low frequencies for evoked activity.

Fig. 7 provides an estimate of the improvement in SNR with either epoch-based or component-based rejection methods. Data had been previously preprocessed with SSS. Subsequently, only one of the following five rejection types was applied: epoch-based rejection targeting jumps, muscle or eye artifacts or componentbased rejection employing ICA or SOBI. Epoch-based rejection

Table 2

Mean SNR across recordings with and without continuous head position monitoring before and after preprocessing with SSS and tSSS.

\begin{tabular}{lll}
\hline & With head coils & Without head coils \\
\hline Unprocessed & 2.2 & 2.0 \\
SSS & 3.7 & 4.3 \\
tSSS & 3.8 & 4.1 \\
\hline
\end{tabular}

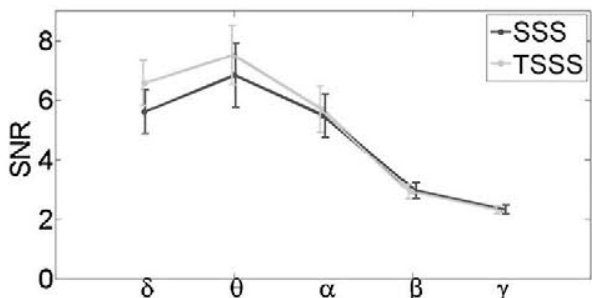

Fig. 5. Evoked activity. Mean and standard error of the SNR in the different frequency bands after applying SSS and tSSS.

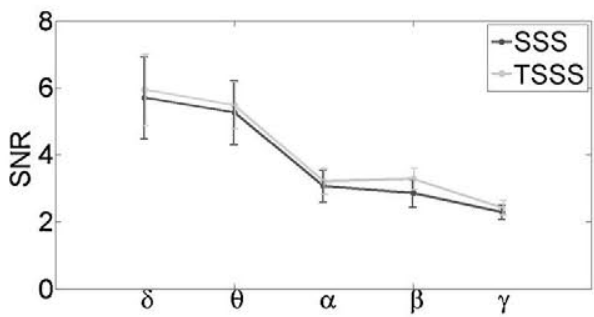

Fig. 6. Induced activity. Mean and standard error of the SNR in the different frequency bands after applying SSS and tSSS.

provides approximately a 5\% increase for jumps and muscle artifacts and around a $10 \%$ for eye artifacts. The improvement is clearly higher, around $35 \%$, for ICA and SOBI.

Finally Fig. 8 displays changes in SNR as a function of the fraction of epochs rejected after identifying the three different types of artifacts. Rejection was carried out by defining a threshold that would separately reject a given fraction of $z$-values for ocular, muscular and jump artifacts. Epochs with one or more artifacts were rejected. While for all previous results epochs corresponding to the encoding phase of the first experimental condition were 


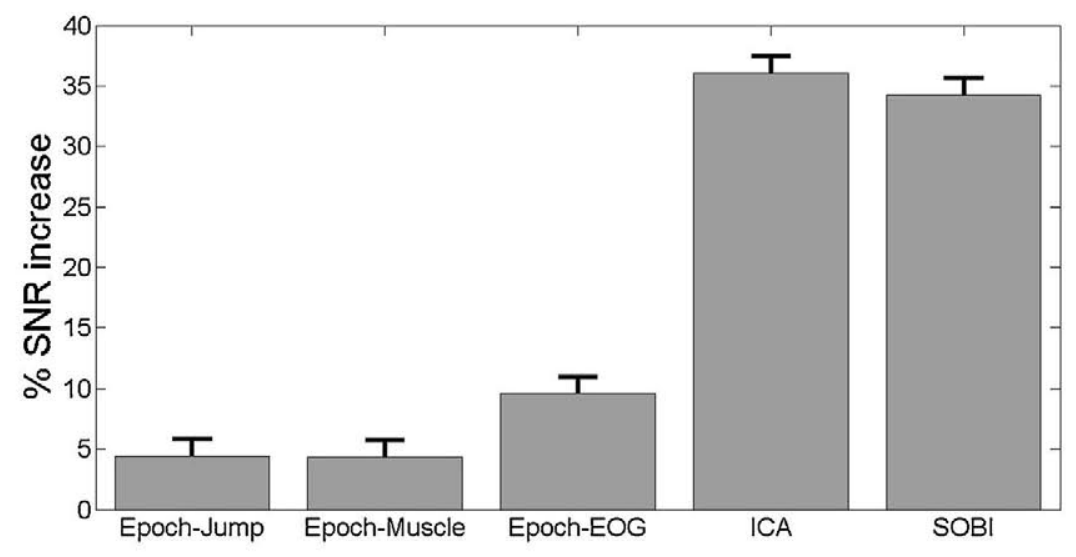

Fig. 7. Increase in SNR after applying epoch and component-based artifact rejection.

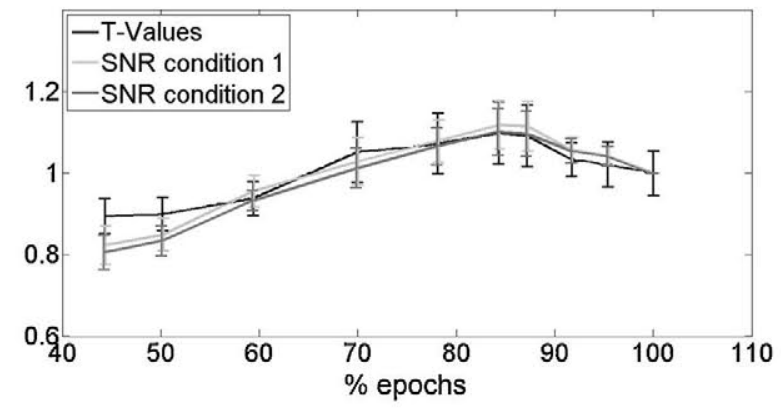

Fig. 8. SNR vs, fraction of non-rejected epochs for the 2 experimental conditions and statistical difference (Student $t$-value) between the two conditions. Mean and s.e.m. across 10 recordings. Curves are normalized by dividing by the value at $100 \%$.

considered, in the present Figure curves for condition 1 and 2 for the recognition phase are represented. In addition, the $t$-values corresponding to an unpaired $t$-test across trials between the two conditions are reported for comparison. An artifact reduction procedure which finds a good compromise between rejecting artifacts and preserving signal should also provide an increase in the statistical difference between independent experimental conditions since artifacts affect both conditions in a similar fashion. Curves and errorbars denote the mean and the standard error of the mean across 10 representative recordings. Curves are normalized by dividing by the value at $100 \%$. All three measures provide a similar estimate of an optimal rejection rate of about $10-15 \%$ of epochs.

\section{Discussion}

In the present work we evaluated the effect of a number of preprocessing methods on the signal-to-noise ratio of the MEG signal. First-step methods SSS and tSSS provided a SNR increase of around $100 \%$ for broadband signals. Both methods yielded also similar SNRs after bandpass-filtering the data in the traditional frequency bands, both for induced and evoked activity. Epoch rejection algorithms increased the SNR by $5-10 \%$. Finally, component-based methods yielded an improvement close to $35 \%$. The highest increase in SNR occurred when the fraction of rejected epochs was around $10 \%$. This is also the rejection rate which maximizes the statistical difference between experimental conditions.

The application of a method to reduce environmental noise such as SSS or tSSS in Elekta systems, or reference-based methods in MEG scanners from other manufacturer is mandatory to obtain usable data. In the present analysis both SSS and tSSS provided similar results. Nevertheless tSSS has been shown to be superior for particularly noisy datasets like those contaminated by contributions from dental braces and fillings, metal implants or stimulators (Song et al., 2009; Jin et al., 2013) which were not considered in the present analysis. If no such recordings are anticipated SSS may have the advantage of implying less data processing, which is to be preferred for similar results. Component-based methods were superior to epoch-based rejection. While the latter are routinely used the former are less frequently employed. The present results suggest that the use of component-based artifact reduction algorithms is advisable. Both types of methods can be used in parallel. Similar results in terms of SNR were found with and without continuous head position monitoring, which indicates that the high-frequency signal employed to locate the head did not adversely affect the recorded signal originating from the brain. Therefore head position monitoring seems advisable if head movements are foreseen.

The SNR here defined exploits the fact that a response is evoked/induced at experimentally controlled times. This allows distinguishing brain responses that are phase-locked or timelocked to the stimulus from artifacts independent from the stimulus, which contribute to the variability of the signal, but not to the amplitude of the signal mean. Such an approach is not possible for resting-state paradigms. In the absence of a stimulus or task we can quantify the reproducibility of the signal, but we cannot rule out contributions from recurring artifacts. Even if the present analysis does not include resting-state data, the conclusions on the effectiveness of the different algorithms also apply to these paradigms as the artifacts do not depend on the paradigm employed.

While the present work assessed the effect of the different preprocessing methods on the signal-to-noise of the event-related fields, it is likely that the methods that provide the largest SNR increase are also those for which the resulting single-trials are more artifact-free, as artifacts tend to reduce the ERF's SNR. In fact, the method developed in Talsma (2008) is based on selecting singletrials according to their impact on the ERF's SNR.

In the present analysis the global SNR was calculated by averaging over the $20 \%$ of samples with highest local SNR within the window of interest. Alternative definitions where, for example, channels of interest were selected, yielded similar results to the present ones. Results using the statistical difference between experimental conditions were also similar. Therefore the SNR definition used seems to be a sensible measure of the effectiveness of preprocessing in reducing artifacts while preserving the signal originating from the brain. Preliminary analysis identified similar behavior in terms of SNR changes for magnetometers and planar gradiometers, and for that reason we focused on magnetometers. Possible extensions of the present work include considering additional sensor types such as axial gradiometers as well as estimations of source activity from source reconstruction algorithms (Baillet 
et al., 2001; Darvas et al., 2004; Zumer et al., 2008). Different preprocessing methods or combinations of them could also be considered. Calculating the SNR of datasets may also be of use to decide which datasets are too noisy and need to be discarded from further analysis. In conclusion, assessing the Signal-to-noise ratio may help to quantify the quality of both the preprocessing stages and the recordings.

\section{Acknowledgment}

We acknowledge funding from Grant PSI2010-22118 from the Spanish Ministry of Science and Innovation to Angel Nevado.

\section{References}

Baillet S, Mosher JC, Leahy RM. Electromagnetic brain mapping. Signal Processing Magazine, IEEE 2001;18(6):14-30.

Belouchrani A, Abed-Meraim K, Cardoso J-F, Moulines E. A blind source separation technique using second-order statistics. IEEE Transactions on Signal Processing 1997:45(2):434-44.

Cardoso J-F. Blind signal separation: statistical principles. Proceedings of the IEEE 1998:86(10):2009-25.

Cichocki A, Shishkin SL, Musha T, Leonowicz Z, Asada T, Kurachi T, Eeg filtering based on blind source separation (bss) for early detection of alzheimer's disease. Clinical Neurophysiology 2005:116(3):729-37.

Comon P. Independent component analysis, a new concept? Signal Processing 1994;36(3):287-314

Darvas F, Pantazis D, Kucukaltun-Yildirim E, Leahy R. Mapping human brain function with meg and eeg: methods and validation. Neurolmage 2004:23:S289-99, 13 .

Gross J, Baillet S, Barnes GR, Henson RN, Hillebrand A, Jensen O, et al. Good practice for conducting and reporting MEG research. Neurolmage 2013;65:349-63.

Hamalainen M, Hari R, Ilmoniemi RJ, Knuutila J, Lounasmaa OV. Magnetoencephalographytheory, instrumentation, and applications to noninvasive studies of the working human brain. Reviews of Modem Physics 1993;65(2):413-97.

Hari R, Salmelin R. Magnetoencephalography: from squids to neuro-science: neuroimage 20th anniversary special edition. Neurolmage 2012;61(2):386-96.

Hu I, Mouraux A, Hu Y, Iannetti GD. A novel approach for enhancing the signal-tonoise ratio and detecting automatically event-related potentials (erps) in single trials. Neurolmage 2010;50(1):99-111.
Hyvrinen A, Oja E. Independent component analysis: algorithms and applications. Neural Networks 2000;13(45):411-30.

Jin $\mathrm{K}$, Alexopoulos AV, Mosher JC, Burgess RC. Implanted medical devices or other strong sources of interference are not barriers to magnetoencephalographic recordings in epilepsy patients. Clinical Neuro-physiology 2013;124(7): $1283-9$.

Leonowicz Z, Karvanen J, Shishkin SI. Trimmed estimators for robust averaging of event-related potentials. Journal of Neuroscience Methods 2005;142(1): $17-26$.

Nolan H, Whelan R, Reilly R. Faster: fully automated statistical thresholding for eeg artifact rejection. Journal of Neuroscience Methods 2010;192(1):152-62.

Nolte G, Curio G. The effect of artifact rejection by signal-space projection on source localization accuracy in MEG measurements. IEEE Transactions on Biomedical Engineering 1999:46(4):400-8.

Oostenveld R, Fries P, Maris E, Schoffelen J-M. Fieldtrip: open source software for advanced analysis of meg, eeg, and invasive electrophys-iological data. Computational Intelligence and Neuroscience 2011;2011:156869.

Parkkonen L. Instrumentation and data preprocessing. In: Hansen P, Kringelbach M, Salmelin R, editors. MEG: An Introduction to Methods. New York: Oxford University Press; 2010. p. 75-82.

Song T, Cui I, Gaa K, Feffer L, Taulu S, Lee RR, et al. Signal space separation algorithm and its application on suppressing artifacts caused by vagus nerve stimulation for magnetoencephalography recordings. Journal of Clinical Neurophysiology 2009;26(6):392-400.

Talsma D. Auto-adaptive averaging: detecting artifacts in event-related potential data using a fully automated procedure. Psychophysiology 2008;45(2):216-28.

Tang AC, Sutherland MT, McKinney C. Validation of SOBI components from high density EEG. Neurolmage 2005:25(April (2)):539-53.

Taulu S, Kajola M, Simola J. Suppression of interference and artifacts by the Signal Space Separation Method. Brain Topography 2004;16(4):269-75.

Taulu S, Simola J. Spatiotemporal signal space separation method for rejecting nearby interference in meg measurements. Physics in Medicine and Biology 2006:51(7):1759-68.

Taulu S, Simola J, Kajola M. Applications of the signal space separation method, IEEE Transactions on Signal Processing 2005:53(9):3359-72.

Uusitalo MA, Ilmoniemi RJ. Signal-space projection method for separating MEG or EEG into components. Medical \& Biological Engineering \& Computing 1997; 35(2): 135-40.

Vrba J, Robinson S. Signal processing in magnetoencephalograpy. Methods 2001:25:249-71.

Zumer JM, Attias HT, Sekihara K, Nagarajan SS. Probabilistic algorithms for meg/eeg source reconstruction using temporal basis functions learned from data. Neurolmage 2008;41(3):924-40. 